\title{
Strömgren-H $\beta$ photometry of the young open cluster NGC 6913
}

\author{
Nadia Kaltcheva ${ }^{1}$, Valeri Golev ${ }^{2}$, John Beaver ${ }^{3}$, Steven Lund ${ }^{1}$ and \\ Michael Briley ${ }^{4}$ \\ ${ }^{1}$ Department of Physics and Astronomy, University of Wisconsin Oshkosh, \\ 800 Algoma Blvd., Oshkosh, WI 54901, USA \\ email: kaltchev@uwosh.edu \\ ${ }^{2}$ Department of Astronomy, Faculty of Physics, St Kliment Ohridski University of Sofia, \\ 5 James Bourchier Blvd., BG-1164 Sofia, Bulgaria \\ email: valgol@phys.uni-sofia.bg \\ ${ }^{3}$ University of Wisconsin - Fox Valley, 1874 Midway Rd, Menasha, WI, USA \\ email: john. beaver@uwc.edu \\ ${ }^{4}$ Department of Physics and Astronomy, Appalachian State University, Boone, NC 28608, \\ USA \\ email: brileymm@appstate.edu
}

\begin{abstract}
We present new CCD uvby $\beta$ photometry of about 50 stars located in the central region of the open cluster NGC 6913. The individual stellar distances and color excesses of established cluster's members are analyzed and new estimate of the cluster's distance is provided.
\end{abstract}

Keywords. Stellar clusters and associations, uvby $\beta$ photometry, NGC 6913.

\section{Introduction}

The young open cluster NGC 6913 (M 29) is located in a low-extinction area toward the Great Cygnus Rift. Despite several extensive studies, the distance estimates to the cluster are still controversial, differing by as much as a factor of two. Tifft (1958) suggested two groups at 1.6 and $2.1 \mathrm{kpc}$. Photoelectric uvby $\beta$ photometry yielded $1.1 \mathrm{kpc}$, not in agreement with spectroscopically-based estimates (Crawford et al. 1977). More recently, utilizing Vilnius, BV and JHK photometry and spectra, Straizys et al. (2014) obtained $1.54 \mathrm{kpc}$. In order to re-evaluate the main cluster parameters, we provide new analysis of the existing uvby $\beta$ data and supplement them with new CCD uvby $\beta$ photometry of the central $15 \times 15$ arcmin region of NGC 6913 . The uvby $\beta$ photometric system (Strömgren 1966, Crawford \& Mander 1966) should in general be able to provide independent estimates of reddening, distance, metallicity and age, thus helping to constrain the existing disagreements.

\section{Observations}

The data were obtained during three consecutive nights in May/June, 2008, on the 0.9 m WIYN (Wisconsin Indiana Yale National Optical Astronomy Observatory) telescope at Kitt Peak National Observatory (KPNO). The telescope was equipped with the S2KB CCD camera, which employs a $2048 \times 2048,21 \mu$-pixel detector for a field of view of 20.5 arcmin, and a set of KPNO uvby $\beta$ filters. The reduction of the images followed standard procedures for bias subtraction, flat fielding, illumination and scattered light corrections 
using the IRAF software package. About 20 cluster's stars with uvby $\beta$ photoelectric photometry (Crawford et al. 1977) were used as standards. The reduction was carried out in $V, b-y, c_{1}, m_{1}$ and $\beta$, as outlined by Crawford \& Barnes (1970). In order to account for an apparent second-order variations in the point spread function across the field, we investigated various approaches to perform the photometry, including aperture photometry and 2-dimensional fitting with Gauss and Moffat functions. The 2-dimensional Gauss fit was found to provide the least error in the photometric transformations.

\section{Results and Discussion}

Our analysis of the photoelectric uvby $\beta$ data of Crawford \& Barnes (1970) indicates that the bright stars that make the cluster recognizable are located at a distance of $895(+47,-44)$ pc. The fainter stars in the field seem to be more distant by $500 \mathrm{pc}$.

The new CCD uvby $\beta$ observations support this conclusion. Up to a limiting magnitude of about 14, we found no B-type stars located closer than $1 \mathrm{kpc}$. The impression is that the young compact group of bright $\mathrm{OB}$ stars is projected onto a background of fainter B-type stars. The obtained distance of $895 \pm 45 \mathrm{pc}$ is significantly smaller than the spectroscopically based $1540 \pm 150$ pc (Straizys et al. 2014). All these stars are located closer than a distance modulus of $11 \mathrm{mag}$, which corresponds to $1.6 \pm 0.35 \mathrm{kpc}$ (assuming an error of $\pm 0.5 \mathrm{mag}$ in the distance modulus determination). This disagreement arises most likely from a possible overestimation of the spectroscopic distances(details on such comparisons can be found in Kaltcheva \& Golev 2011). The inspection of the photometric diagrams do not imply a peculiar behavior that can significantly affect the uvby $\beta$ photometry-derived distances for the brightest cluster's members.

NGC 6913 is positioned within the boundaries of the Cyg OB1 association, one of the several OB associations within the giant H II region Sh 2-109. This nebulosity is possibly a complex of smaller H II regions overlapping along our line of sight since in this direction we are looking straight down the Orion spur (Sharpless 1959). Recent X-ray emission findings (Uyaniker et al. 2001) also suggest that Sh 2-109 consists of overlapping, not physically related, features. A distance less than $1 \mathrm{kpc}$, as suggested here, would place NGC 6913 closer than other young clusters located within the formal boundaries on the Cyg OB1/OB3 associations, like NGC 6871 and IC 4996, implying overlapping star-forming regions in this direction.

Acknowledgements. This study made use of the NASA Astrophysics Data System, SIMBAD database and WEBDA open cluster database operated at the Institute for Astronomy of the University of Vienna. N.K. acknowledges support from the SNC Endowed Professorship at the University of Wisconsin Oshkosh. This work is supported by the National Science Foundation grant AST-1516932.

\section{References}

Crawford, D. L. \& Mander, J. 1966, AJ, 71, 114

Crawford, D. L. \& Barnes, J. V. 1970, AJ, 75, 978

Crawford, D. L., Barnes, J. V., \& Hill, G. 1977, AJ, 82, 606

Kaltcheva, N. \& Golev, V. 2011, Stellar Clusters \& Associations, A RIA Workshop on Gaia Proceedings, p. 299

Sharpless, S. 1959, ApJ Suppl., 4, 257

Straizys, V., Milasius, K., \& Boyle, P., et al. 2014, AJ, 455, 850

Strömgren, B. 1966, ARAA, 4, 433

Tifft, W. G. $1958, A J, 63,127$

Uyaniker, B., Fürst, E., Reich, W., Aschenbach, B., \& Wielebinski, R. 2001, A\&̊A, 371, 675 